\title{
Host-Specific Generation and Maintenance of Tomato bushy stunt virus Defective Interfering RNAs
}

\author{
Rustem T. Omarov, Jorge A. M. Rezende, and Herman B. Scholthof \\ Department of Plant Pathology and Microbiology, Texas A\&M University, College Station 77843, U.S.A.
}

Submitted 2 June 2003. Accepted 6 October 2003.

\begin{abstract}
The accumulation of Tomato bushy stunt virus (TBSV) defective interfering RNAs (DIs) has been observed in several species of plants, but the involvement of host-specific processes and the functional role of DIs are still poorly understood. In this study, the accumulation of DIs was compared after several passages of TBSV through Nicotiana benthamiana and pepper (Capsicum annuum). As anticipated, passages of wild-type TBSV through $N$. benthamiana resulted in the accumulation of significant levels of TBSV DIs, which caused symptom attenuation and prevented the plants from lethal necrosis. On the contrary, TBSV infection of pepper plants caused severe local and systemic chlorosis, but continuous virus passages did not result in detectable levels of DIs accumulation. In addition, the inoculation of pepper plants with a mixture of helper virus and DI either from in vitro generated transcripts or from infected $N$. benthamiana did not yield DI in upper pepper leaves. Our cumulative results suggest that complex host-specific determinants play an important role in TBSV DI generation and their subsequent maintenance and accumulation.
\end{abstract}

Tomato bushy stunt virus (TBSV), the type member of the genus Tombusvirus in the family Tombusviridae is a widely distributed soil-borne root pathogen that replicates to high titers in about 100 plant species in 20 different families (Martelli et al. 1988). TBSV particles encapsidate a single copy of a positivesense single-stranded RNA (ssRNA) genome of approximately 4,800 nucleotides (nt) (Hearne et al. 1990). The genomic RNA (gRNA) functions as an mRNA for the translation of the two 5'-proximal genes, encoding a $33-\mathrm{kDa}$ protein (P33) and a readthrough product of $92 \mathrm{kDa}$ (P92) (Fig. 1); both are required for replication (Scholthof et al. 1995a).

The 41-kDa TBSV coat protein (CP) gene is translated from subgenomic RNA1 (sgRNA1) (Hillman et al. 1989) and is required in a host-specific manner for efficient systemic spread (Desvoyes and Scholthof 2002; Scholthof et al. 1993; Turina et al. 2003). Two nested genes, $p 22$ and $p 19$, located near the 3 ' terminus of the gRNA, are translated from subgenomic RNA2 (sgRNA2) (Johnston and Rochon 1990). These genes express proteins of $22 \mathrm{kDa}$ (P22) and $19 \mathrm{kDa}$ (P19), respectively (Johnston and Rochon 1996; Rochon and Johnston 1991; Scholthof et al. 1995b).

P22 is a typical membrane-associated cell-to-cell movement protein (Chu et al. 1999), while the cytosolic P19 is involved

Corresponding author: H. B. Scholthof; Telephone: (979) 862-1495; Fax: (979) 845-6483; E-mail: herscho@tamu.edu

Current address of J. A. M. Rezende: Departamento de Entomologia, Fitopatologia e Zoologia Agricola, Escola Superior de Agricultura, 'Luiz de Queiroz', Universidade de São Paulo, Piracicaba, SP13418, Brazil. in various biological activities. For example, although P19 is dispensable for infection of the laboratory hosts Nicotiana benthamiana and $N$. clevelandii, it is essential for efficient systemic infection in spinach (Spinacia oleracea) and pepper (Capsicum annuum) as well as for localized infections on cowpea (Vigna unguiculata) (Scholthof et al. 1995b, 1999; Turina et al. 2003). Moreover, P19 elicits a hypersensitive response (HR) on N. tabacum, and it induces systemic necrosis in $N$. benthamiana (Scholthof et al. 1995c). Recently, it was shown that P19 is a strong suppressor of post-transcriptional gene silencing (PTGS) and a moderate suppressor of virus-induced gene silencing (Qiu et al. 2002; Voinnet et al. 1999).

Defective interfering RNAs (DIs) have been documented in several positive-sense ssRNA virus groups and represent one of several symptom-modulating RNAs identified in association with plant virus RNA infections (Roux et al. 1991; Russo et al. 1994; White and Morris 1999). Tombusviruses are well known for their ability to produce DIs that are subsequently trans-replicated to high levels by the helper virus (Hillman et al. 1987; Knorr et al. 1991). Structurally, Tombusvirus DIs are deletion mutants that represent truncated forms of the viral genome that have lost functions essential for independent replication. The accumulation of TBSV DI in protoplasts depends exclusively on the P33 and P92 replicase proteins (Scholthof et al. 1995d). Molecular studies of various Tombusvirus DIs exhibit their broad diversity in size and sequence but, in essence, they are composed of a portion of the $5^{\prime}$ terminus, an internal region of $p 92$, and various portions of the $3^{\prime}$ terminus of the viral genome (Burgyan et al. 1991; Hillman et al. 1987; Knorr et al. 1991; White and Morris 1994). The distinct segments of gRNA that make up these DIs are denoted as regions I through IV (Fig. 1) (White and Morris 1999). Regions II and III are variable, while regions I and IV are conserved (White and Morris 1994).

DI do not normally accumulate to readily detectable levels during natural infections (Celix et al. 1997), despite their rapid accumulation under laboratory conditions in $N$. benthamiana and $N$. clevelandii (Knorr et al. 1991). In Nicotiana hosts that support TBSV infections, a systemic necrosis rapidly develops in plants infected with virus isolates lacking DIs. However, plants infected with TBSV in the presence of DIs develop an attenuated mosaic (Scholthof et al. 1995d). Tombusvirus DIs reduce the accumulation of helper virus RNA in protoplasts as well as in plants (Chang et al. 1995), since they somehow interfere with normal replication of the parental virus from which they are derived (Hillman et al. 1987; Jones et al. 1990; Qiu et al. 2001; Scholthof et al. 1995d). Modulation of symptoms could be related to DI-mediated reduction of necrogenic P19 accumulation (Scholthof et al. 1995d). Furthermore, recent studies suggest that PTGS may play an important role in 
the selective accumulation of DIs and their symptom attenuation (Qiu et al 2002; Szittya et al. 2002).

To examine the effect of the host on TBSV infection, we routinely use pepper, since this host provides clear contrasts with $N$. benthamiana regarding TBSV infection. Some of these comparisons appear particularly relevant for the present study, because they raised questions about possible involvement of host-specific determinants in the formation of DIs. For example, infections of pepper with TBSV inflict stunting and severe chlorosis, yet the lethal necrosis typically induced in $N$. benthamiana does not occur in pepper (Scholthof et al. 1995b). This would suggest that the activity of DIs to protect against a lethal necrosis would be superfluous in pepper, and thus, their presence would serve no protective function for this host. Furthermore, intermolecular $\mathrm{CP}$ mutant recombinants frequently accumulate in $N$. benthamiana but not in pepper (Desvoyes and Scholthof 2002). This raised the question whether recombination events responsible for DI generation might also be under host-specific control. Lastly, since P19 is required for spread in pepper and DIs substantially interfere with accumulation of P19 (Scholthof et al. 1995d), the implication is that maintenance of DIs could have a negative impact on the ability of TBSV to infect pepper.

The objective of the present study was to compare TBSV infections of pepper with those of $N$. benthamiana with regards to de novo DI generation, accumulation, and maintenance and their effect on symptom development. The results showed that, in contrast to rapid generation and accumulation of DIs in $N$. benthamiana, continuous TBSV passages through pepper did not result in DI accumulation. Furthermore the inoculation
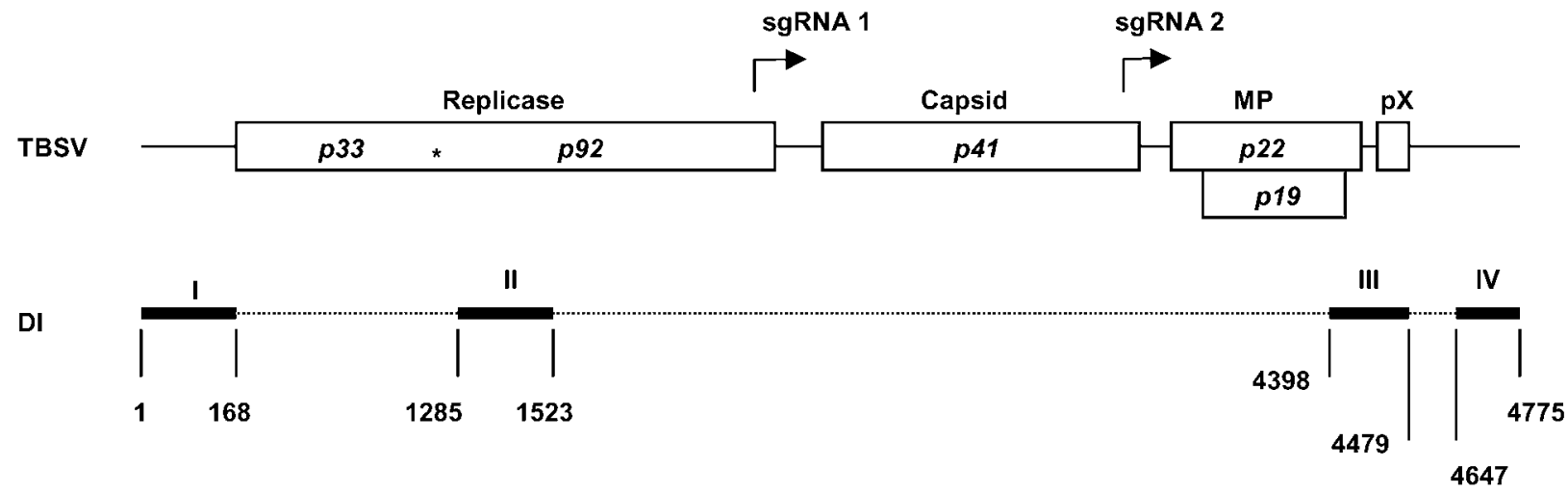

Fig. 1. Schematic diagrams of the Tomato bushy stunt virus (TBSV) genome and defective interfering RNA (DI). Open boxes denote open reading frames (ORF) of the encoded proteins (in $\mathrm{kDa}$ ) for which functions are indicated above the boxes. MP indicates movement proteins expressed from overlapping $p 22$ and $p 19$ ORF. The function of $p X$ is unknown, but its RNA sequences have host-dependent effect on viral RNA accumulation (Scholthof and Jackson 1997). The solid lines between ORF indicate presumed untranslated regions. The transcription start sites for sgRNA1 and sgRNA2 are indicated. The asterisk inside the TBSV genome indicates the readthrough amber stop codon of $p 33$ for expression of $p 92$. Regions I through IV represent the conserved motifs present in DIs. The dashed lines between these regions indicate sequences that are deleted from the TBSV genome during DI formation. The nucleotide positions are based on an infectious cDNA clone of the wild-type genome, pTBSV-100 (Hearne et al. 1990) and a typical infectious DI cDNA, B10 (Knorr et al. 1991).

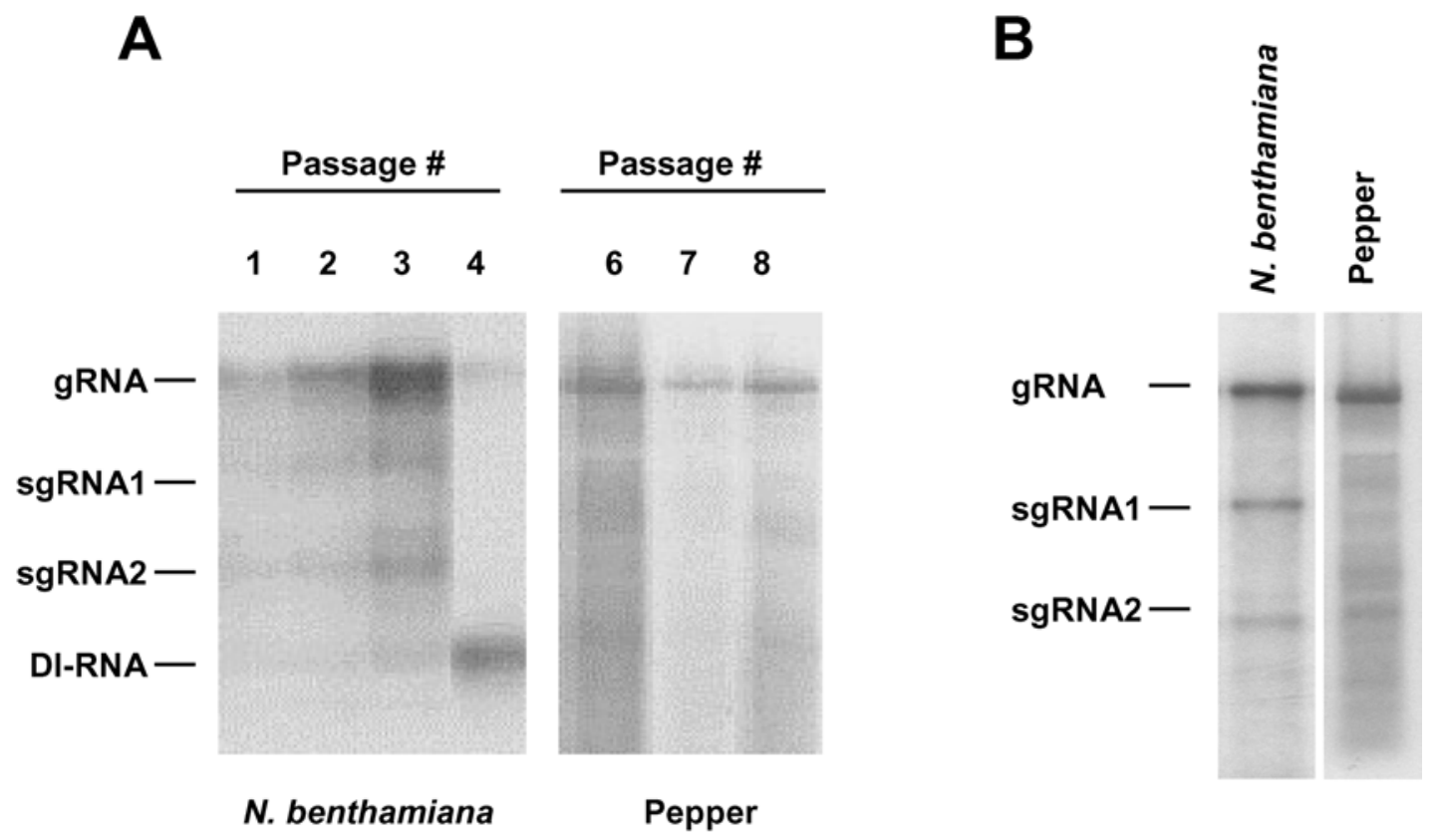

Fig. 2. Tomato bushy stunt virus (TBSV) defective interfering RNA (DI) generation in Nicotiana benthamiana and pepper plants after virus passages. A, For the first passage, plants were inoculated with in vitro synthesized TBSV transcript. Hybridization was performed with a ${ }^{32} \mathrm{P}-\mathrm{labeled}$ probe representing the full-length TBSV. The positions of genomic (gRNA), subgenomic (sgRNA1, sgRNA2), and DI are marked. Numbers on the top indicate passages of TBSV RNA. For pepper plants, only final passages are shown. DIs were not observed at earlier stages (not shown). B, Differences in pattern of TBSV gRNA, sgRNA1, and sgRNA2 extracted from $N$. benthamiana and pepper. Plants were inoculated with in vitro-synthesized TBSV transcripts, and hybridization was performed with a ${ }^{32} \mathrm{P}$-labeled probe representing the full-length TBSV RNA. 
of pepper plants with inoculum mixtures of helper virus and DI did not yield any detectable levels of DI accumulation in upper leaves of pepper. These results support the hypothesis that the host plant plays an important role in the mechanism of DI generation and maintenance.

\section{RESULTS}

Inability of TBSV to generate DIs in pepper.

As expected, TBSV passages through $N$. benthamiana plants reproducibly resulted in formation and accumulation of readily detectable levels of DIs upon the fourth consecutive passage (Fig. 2A). The passages of TBSV and the resulting accumulation of DIs caused symptom attenuation and prevented the plants from lethal necrosis, as documented in other studies (Hillman et al. 1987; Russo et al. 1994; White and Morris 1999). In parallel experiments, we analyzed DI accumulation in pepper plants. For this purpose, we first conducted multiple passages of TBSV, which is a routine procedure toward acquiring a DI-containing population. Continuous passages through pepper were performed up to eight times, but this consistently failed to result in detectable levels of TBSV DI accumulation (Fig. 2A).

Using full-length TBSV cDNA as a probe, TBSV gRNA, sgRNA1, and sgRNA2 were readily detectable by Northern blot hybridization from total RNA extracted from $N$. benthamiana plants. However, with the same probe, RNA samples

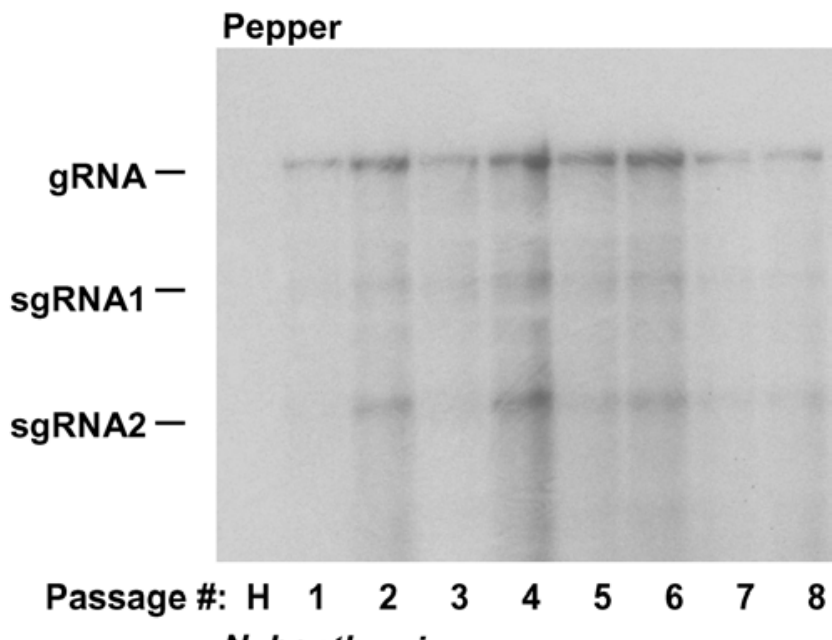

N. benthamiana

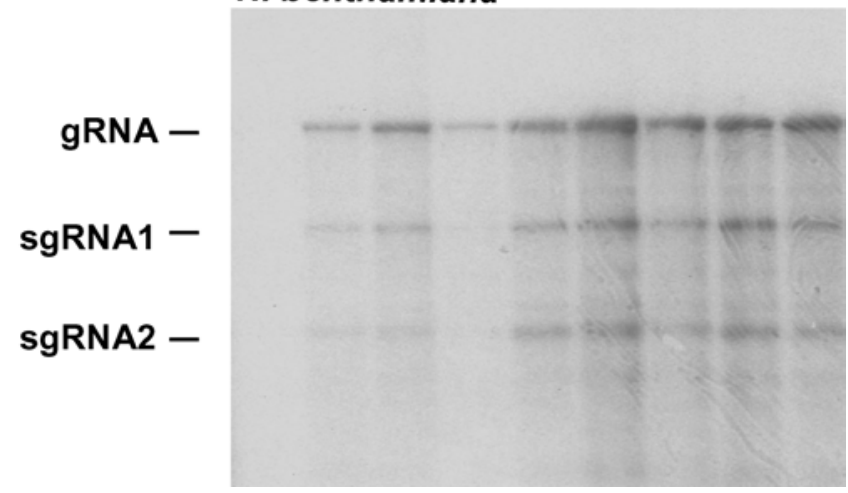

Fig. 3. Passages of Tomato bushy stunt virus (TBSV) through pepper plants and coinoculation of Nicotiana benthamiana. Each $N$. benthamiana plant was infected with plant sap from a different passage through pepper. Pepper plants in passage 1 were inoculated with in vitro-synthesized TBSV transcripts. Hybridization was performed with a ${ }^{32} \mathrm{P}$-labeled probe specific for the $3^{\prime}$ terminal 890 nt of TBSV RNA (pBW-1). Numbers on the top indicate passages of TBSV. The positions of genomic (gRNA) and subgenomic (sgRNA1, sgRNA2) are marked. extracted from pepper exhibited predominantly genomic viral RNA (Fig. 2B). The presence of sgRNA1 and sgRNA2 in these samples was not readily distinguishable. However, when the blots of total RNA from infected pepper plants were probed with cloned cDNA from the 3'-terminal (890 nt) of TBSV RNA (pBW-1) (Qiu and Scholthof 2001), the sgRNA1 and sgRNA2 were detected. Yet even with this more sensitive system, detectable levels of DIs were still not observed in the analyzed samples (Fig. 3). These results were concordant with the symptomatology, since the TBSV DI coinfected plants did not reveal any obvious differences in symptoms compared with plants inoculated only with TBSV (data not shown). The results in Figure 2B also confirm that TBSV gRNA accumulates to similar levels in both hosts.

To examine the possibility that potentially low amounts (not detectable by Northern blot hybridization) of TBSV DIs were formed in pepper plants, additional tests were performed. Following each of the sequential passages (passages 1 to 8 ) the infected pepper leaves were harvested and macerated for inoculation onto DI-indicator $N$. benthamiana plants. The presence of small amounts of DI in pepper would presumably result in high accumulation of defective molecules in "DI-propagative" $N$. benthamiana and, hence, prevent plants from a lethal necrosis. However, all inoculated $N$. benthamiana plants suffered severe lethal necrosis 8 days after inoculation (data not shown). Also, Northern blot analysis of RNA samples extracted prior to lethal necrosis of $N$. benthamiana plants did not reveal any detectable levels of TBSV DIs (Fig. 3). Furthermore, grafting of $N$. benthamiana scions onto eighth passageinfected pepper rootstock (as potential low DI concentration supplier) resulted in lethal necrosis of the scions, indicating the presence of TBSV and the absence of DI in pepper rootstock (data not shown).

To obtain additional supportive evidence that DIs failed to accumulate in pepper after virus passages, a reverse transcriptase-polymerase chain reaction (RT-PCR) analysis was implemented. RNA was extracted from infected plants and used as template for RT-PCR with specific primers ( $5^{\prime} \mathrm{TBSV}$ primer and 3' TBSV primer [Knorr et al. 1991]). Implementation of this test on $N$. benthamiana plants yielded a DI-specific PCR product, while for pepper plants, the corresponding DNA fragment was not amplified (Fig. 4). The upper PCR-amplified band (approximately $750 \mathrm{bp}$ ) is a serendipitous product of amplification from the genomic TBSV RNA since: i) the same molecular weight DNA fragment was amplified in all TBSVinfected plants ( $N$. benthamiana, pepper), ii) no product was amplified for samples extracted from mock-inoculated plants, iii) this DNA fragment hybridized to TBSV-specific probe

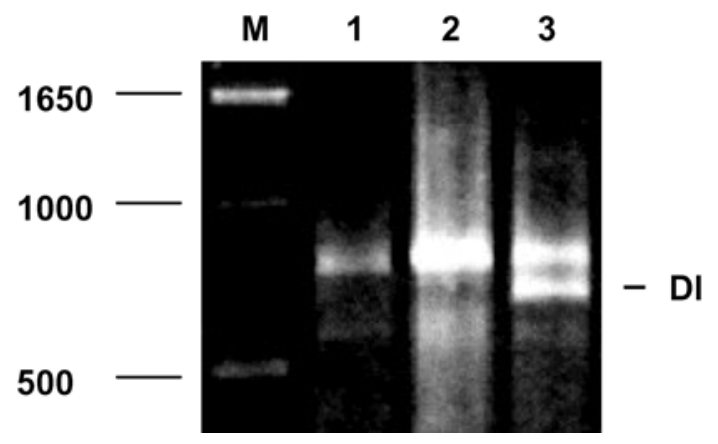

Fig. 4. Reverse transcriptase-polymerase chain reaction analysis of RNA extracted from Nicotiana benthamiana and pepper infected with Tomato bushy stunt virus (TBSV). First passage of $N$. benthamiana (lane 1), eighth passage of pepper (lane 2), and fourth passage of $N$. benthamiana (lane 3) were used as the source for total RNA extraction. M indicates 1kb Plus DNA ladder (Gibco-BRL, Gaithersburg, MD, U.S.A.). 
(data not shown), and iv) the same molecular mass DNA fragment was amplified in $N$. benthamiana first-passage plants that do not contain DI (Fig. 4).

\section{Inability of TBSV to maintain and accumulate exogenously added DIs in pepper.}

To study the ability of TBSV to maintain exogenously added DIs in pepper, we inoculated plants with a mixture of helper virus and DIs extracted from $N$. benthamiana. The amount of DIs in inoculated leaves of pepper decreased gradually after the inoculation (Fig. 5). By 3 days postinoculation (dpi), the amount of genomic TBSV RNA was reproducibly lower in TBSV+DI-inoculated pepper leaves compared with leaves not coinoculated with DIs. However, this difference mostly disappeared by $6 \mathrm{dpi}$. As a positive control, $N$. benthamiana plants were inoculated with the same samples, and these plants showed an increase in DI that resulted in a decreased level of viral RNA (Fig. 5). This DI accumulation was accompanied by the typical symptom attenuation.

To rule out the possibility that the inoculation of pepper with unprotected DI was less efficient compared with infection with virions, an experiment was simultaneously conducted in which plants were inoculated with nonencapsidated, in vitrosynthesized TBSV and TBSV+DI transcripts. This procedure resulted in infection of pepper, as detected by the accumulation of gRNA (Fig. 6), but again, no DIs accumulated.

\section{DISCUSSION}

Virus-host interactions that lead to disease and mechanisms by which DIs influence such symptoms in infected plants are poorly understood. While Tombusvirus infections cause a lethal necrosis in various hosts, the formation and accumulation of DIs in such infections can lead to attenuated (nonlethal) infections in plants (Russo et al. 1994). We report here that, in contrast to infection in many other susceptible hosts, TBSV infections in pepper are nonlethal and DI accumulation was not observed at detectable levels in this host. DIs have been identified and analyzed for the pepper isolate of TBSV (TBSV-P) (Szittya et al. 2000), but the generation of these defective molecules was reported after multiple passages through a different host ( $N$. clevelandii).

It is well known that DI molecules tend to accumulate significantly only after successive multiple passages at artificially high inoculum concentrations (Knorr et al. 1991). It is believed that such conditions favor a coinfection of cells with both helper and DIs. In this study, multiple TBSV passages through pepper did not result in generation of DIs, neither in inoculated nor in upper leaves. Furthermore, none of the infected pepper plants exhibited any noticeable differences in symptoms. Our results also revealed that in pepper TBSV is "impaired" in its ability to maintain exogenously introduced DIs. The detectable amount of DIs in inoculated leaves of pep-
$3 \mathrm{dpi}$

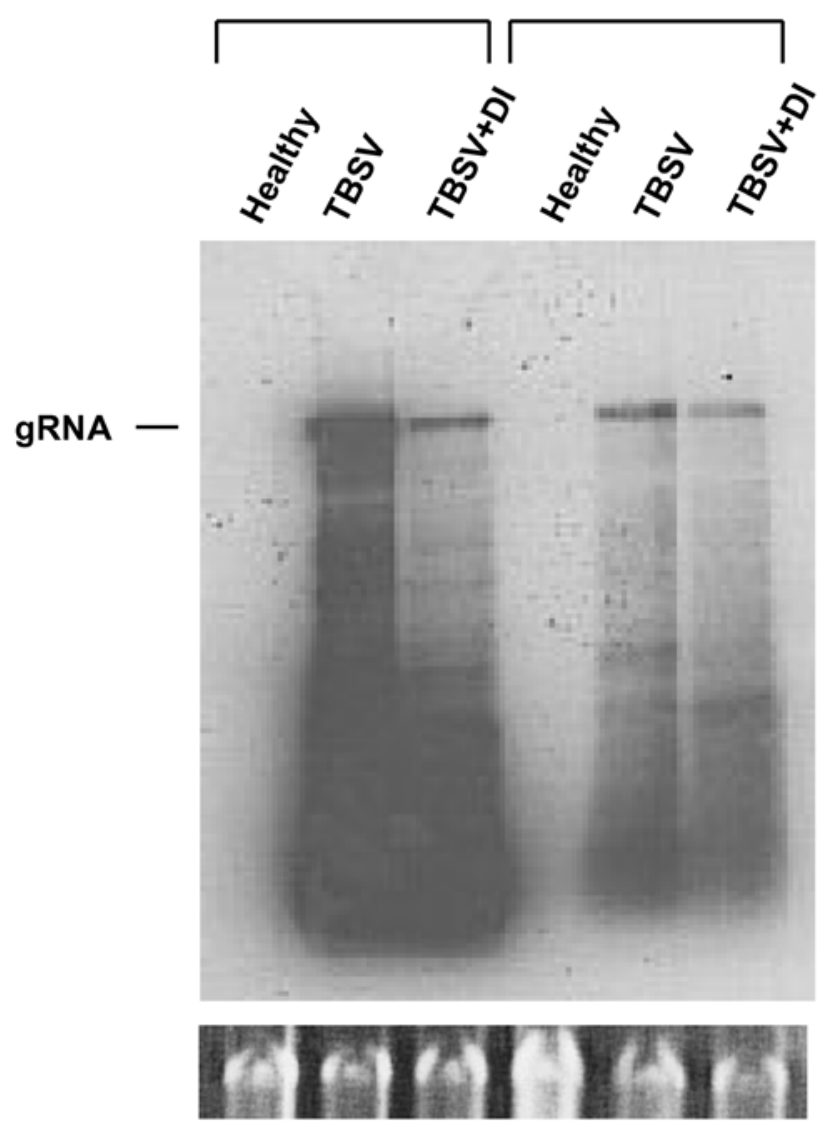

3 dpi

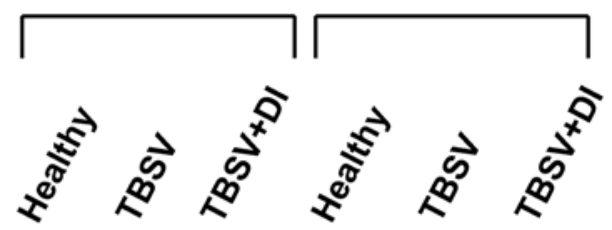

gRNA -

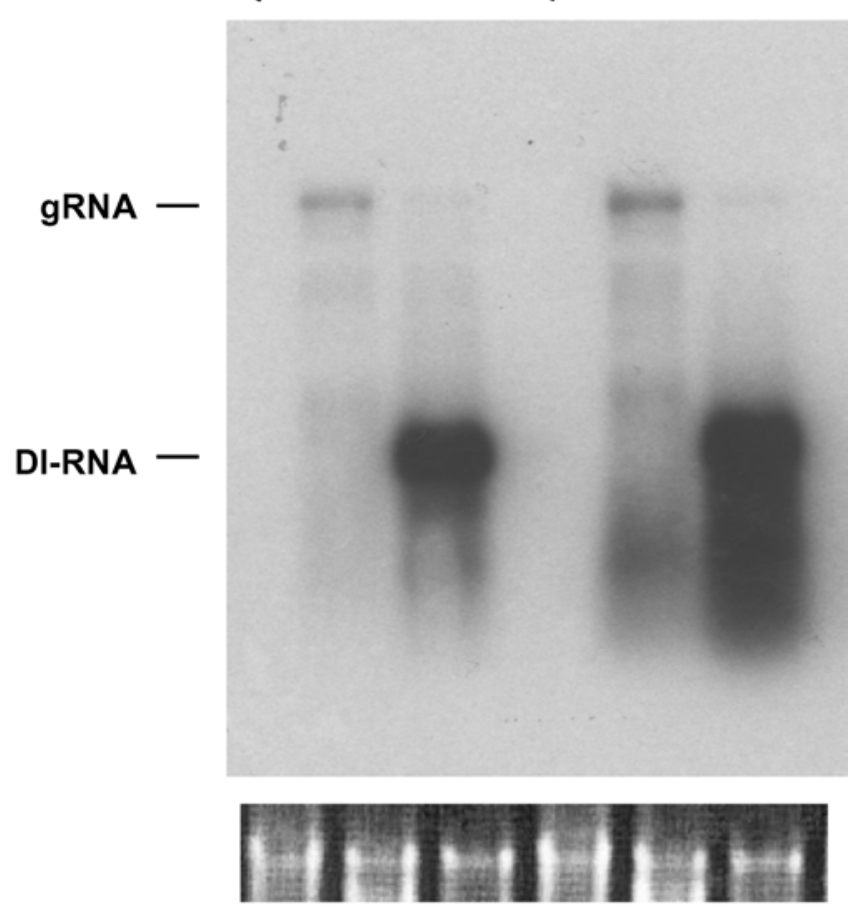

\section{Pepper}

\section{N. benthamiana}

Fig. 5. The amount of Tomato bushy stunt virus (TBSV) defective interfering RNA (DI) in inoculated leaves of pepper and Nicotiana benthamiana at 3 and 6 days postinoculation (dpi). Plants were inoculated with a mixture of in vitro transcripts of genomic TBSV RNA and DI. Hybridization was performed with cDNA clone representing the full-length TBSV RNA. The positions of genomic (gRNA) and DI (DI-RNA) are marked. As control for loading levels, the ethidium bromide-stained gel showing rRNA is provided at the bottom. 
per 3 dpi (Fig. 5) is most likely due to initial high levels of DIs that were inoculated. A drastically decreased amount of DIs at 6 dpi provides supportive evidence that DIs fail to amplify in pepper as compared with $N$. benthamiana. Moreover, no visible symptom differences between plants were detected and no obvious interfering effect on genomic TBSV RNA was evident as compared with $N$. benthamiana plants. Even if DIs were present in pepper plants after consecutive passages but at undetectable levels, still no interfering effect on TBSV gRNA was observed.

It is generally presumed that the mechanism of DI generation in a virus population involves two main events. The first is the formation of the defective viral genome during RNA replication (Perrault 1981; White and Morris 1999). This results in synthesis of an incomplete complementary strand on one template, followed by reinitiation of synthesis on a second template or a different portion of the same template (White and Morris 1994). The next event in DI genesis is its accumulation to a high titer. These separate effects of the host on formation and maintenance of DIs will be discussed in more detail in the following sections.

Viral nonstructural proteins may play an important role in the mechanism of DI accumulation. For example, P22 and P19 might affect the rate of DI accumulation (Rezende et al. 1998; Rochon 1991). Viable DI molecules should also retain cis-acting elements that are necessary for viral replicase recognition as well as signal sequences necessary for movement (Ray and White 1999; White and Morris 1994). However, cis-acting elements that regulate trans-accumulation of replicase-deficient RNAs may be distinct from those controlling replication of the genomic RNA (Qiu et al. 2001). Nevertheless, it has been established that regions II and IV of DIs are essential for replication (Chang et al. 1995), while region I contributes in a strong quantitative manner (Wu and White 1998). Although region III is dispensable, it enhances the competitiveness (Ray and White 1999; White and Morris 1994) and interfering ability of DIs (Chang et al. 1995).

It is possible that in pepper plants the interaction of certain aforementioned viral elements with host-specific determinants required for both DI formation and accumulation of these defective molecules does not occur. Consequently, this may lead to a disrupted ability of TBSV to form and accumulate DI during replication. Alternatively, the lack of DI accumulation may be attributed to the possibility that the process of TBSV replication in pepper occurs under tight cis-preferential regulatory control. This may create conditions that are unfavorable for aberrant events that are necessary for DI generation. The lack of recombination in pepper was also observed for a $\mathrm{CP}$ mutant. This mutant yielded recombinants in N. benthamiana, and although similar rearrangements would be favorable for virus spread in pepper, the recombinants were not generated de novo in this host (Desvoyes and Scholthof 2002). These results suggest that TBSV recombinant events occur in a hostspecific manner.

Examples in which the host influences the composition of DI population have been reported, e.g., passages of a viral inoculum containing DIs through different hosts promote changes in the nature of the dominant population of DIs (Chang et al. 1995). These observations suggest that maintenance of DI is also under host-specific control, which agrees with our present findings on pepper. The responsible mechanism remains speculative, but it may be related to the effect of DIs on proteins expressed from sgRNAs (Scholthof et al. 1995d). It is known that the biological activity of P19 depends on its relative abundance (Scholthof et al. 1999), and it is conceivable that DI-mediated interference with P19 accumulation has serious negative effects on virus spread in this host. Fur- thermore, in pepper, there is no obvious selective advantage associated with maintaining DIs, because no protection from a lethal necrosis is needed. Consequently, the negative effect of DIs on CP and P19 accumulation would, in fact, present a selective disadvantage due to its compromising effect on virus spread. Considering the strict requirements of CP and P19 for movement through pepper (Turina et al. 2003), it is also possible that movement of DIs through pepper is restricted compared with $N$. benthamiana host.

Recently, it was shown that Tombusvirus DIs induce PTGS events that target the helper virus, but DI themselves are somehow protected from the PTGS-associated RNA degradation in N. benthamiana (Qiu et al. 2002; Szittya et al. 2002). PTGSmediated events in pepper could be responsible for an enhanced level of RNA degradation and the reduced levels of sgRNAs (Fig. 2), which, unlike gRNA, are not encapsidated. Within this context, it is possible that, in pepper, the nonencapsidated DIs are degraded by PTGS and thus prevented from accumulation. Whether through activation of PTGS or other mechanisms, the interfering ability of DIs may be a key regulatory element in certain hosts to control the level of TBSV replication. However, as in most plant-virus interactions, the regulatory mechanisms not only depend on the host but surely also on the virus. For example, in the context of DI biogenesis, it is noteworthy that, in contrast to TBSV, Broad bean wilt virus (a Fabavirus) is able to generate and maintain DIs in certain pepper varieties (Lee et al. 2000).

In summary, our results suggest that complex host-specific events may play a crucial role both in formation and accumulation of TBSV DIs in infected tissue. Detailed investigations

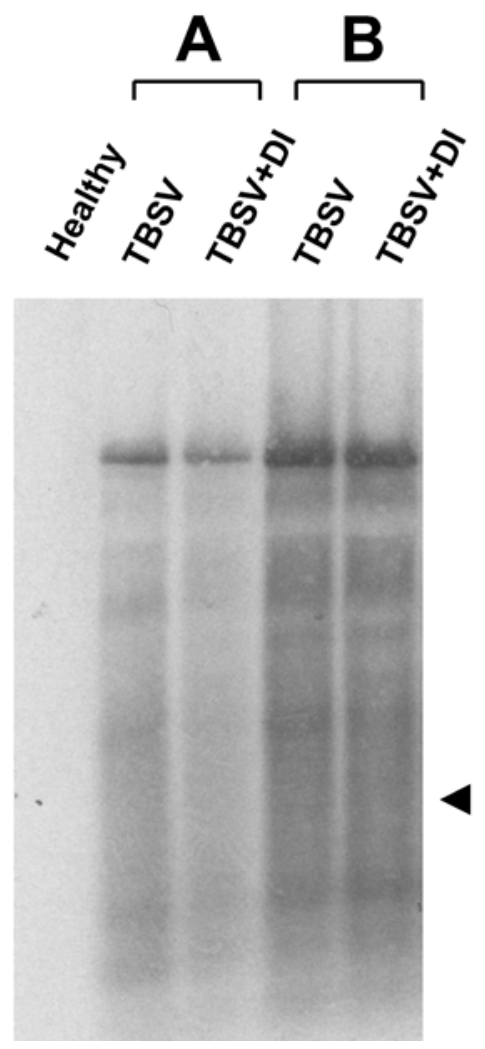

Fig. 6. Northern blot hybridization of RNA extracted from pepper plants (upper leaves). A, Plants infected with a mixture of in vitro synthesized Tomato bushy stunt virus (TBSV) genomic RNA and defective interfering RNA (DI). B, Plants inoculated with plant extracts from infected Nicotiana benthamiana (TBSV, TBSV+DI). Hybridization was performed with a ${ }^{32} \mathrm{P}$-labeled probe representing the full-length TBSV RNA. Plants were analyzed two weeks after inoculation. The arrowhead indicates the position where DI would be expected. 
of host determinants will be necessary to better understand the mechanisms responsible for the separate phases in DI accumulation and their precise roles.

\section{MATERIALS AND METHODS}

\section{Inoculation and analysis of plants.}

In vitro-generated transcripts of TBSV (pTBSV-100) (Hearne et al. 1990) and TBSV DI (B10-18) (Knorr et al. 1991) were prepared essentially as described by Hearne and associates (1990). The TBSV and TBSV DI plasmids $(1 \mu \mathrm{g})$ were linearized at the 3 ' terminus of the viral sequence by digestion with SmaI. Transcript RNAs were synthesized using T7 RNA polymerase and were used for inoculation of plants as previously described (Scholthof et al. 1993). Extracts of transcript-inoculated plants were also used to inoculate plants. For this purpose, plant leaves $(100 \mathrm{mg})$ were homogenized in $1 \mathrm{ml}$ of virus inoculation buffer $\left(0.05 \mathrm{M} \mathrm{KH}_{2} \mathrm{PO}_{4}, 1 \%\right.$ celite $)$.

\section{RNA extraction.}

Total RNAs were extracted by grinding $100 \mathrm{mg}$ of leaf material on ice in $1 \mathrm{ml}$ of extraction buffer $(100 \mathrm{mM}$ Tris- $\mathrm{HCl}$, pH 8.0, $1 \mathrm{mM}$ ETDA, $0.1 \mathrm{M} \mathrm{NaCl}$, and 1\% SDS). Samples were immediately extracted twice with phenol/chloroform $(1: 1, \mathrm{vol} / \mathrm{vol})$ at room temperature and were precipitated overnight with $6 \mathrm{M}$ lithium chloride solution $(1: 1, \mathrm{vol} / \mathrm{vol})$ at $4^{\circ} \mathrm{C}$. The resulting pellets were washed with $70 \%$ ethanol and then resuspended in RNase-free distilled water and were used for Northern blot hybridization and other assays.

\section{Northern blot hybridization.}

RNA extracted from plants was separated on $1.2 \%$ agarose gel in $1 \times$ Tris-borate-EDTA (TBE) (Sambrook et al. 1989). Equal loading of samples was verified by ethidium bromidestaining of the gels. The RNAs were then blotted onto nylon membrane (Osmonics, Promega, Madison, WI, U.S.A.) and were hybridized with a ${ }^{32} \mathrm{P}$-labeled DNA probe corresponding to full-length TBSV RNA, unless otherwise indicated.

\section{RT-PCR.}

RT-PCR analyses using RNA from healthy and infected (TBSV, TBSV+DI) plants were performed with $10 \mu \mathrm{g}$ of total RNA. The oligonucleotides (5' TBSV containing the T7 promoter and 3' TBSV [Knorr et al. 1991]) were added to the reaction mixtures to amplify the TBSV DI sequences. After 30 amplification cycles, the products of PCR reaction were electrophoresed on a $1 \times \mathrm{TBE}$ agarose gel, were blotted, and were hybridized with a ${ }^{32} \mathrm{P}$-labeled DNA probe corresponding to the TBSV RNA sequence.

\section{ACKNOWLEDGMENTS}

We would like to express our thanks to K.-B. G. Scholthof, S. FaureRabasse, and J. Batten for very valuable comments and discussions. Our gratitude to L. Smith for excellent technical assistance. This work was funded by the Texas Agricultural Experiment Station (TEX08387) and grants from the U. S. Department of Agriculture (CSREES-NRI-CGP 9935303-8022), the Texas Higher Education Coordinating Board (ATP 000517-0070-1999), and the National Science Foundation (grant MCB 0131552).

\section{LITERATURE CITED}

Burgyan, J., Rubino, L., and Russo, M. 1991. De novo generation of cymbidium ring spot virus defective interfering RNA. J. Gen. Virol. 72:505509

Celix, A., Rodriguez-Cerezo, E., and Garcia-Arenal, F. 1997. New satellite RNAs, but no DI RNAs, are found in natural populations of tomato bushy stunt tombusvirus. Virology 239:277-284
Chang, Y. C., Borja, M., Scholthof, H. B., Jackson A. O., and Morris, T. J. 1995. Host effects and sequences essential for accumulation of defective interfering RNAs of cucumber necrosis and tomato bushy stunt tombusviruses. Virology 210:41-53.

Chu, M., Park, J.-W., and Scholthof, H. B. 1999. Separate regions on the tomato bushy stunt virus p22 protein mediate cell-to-cell movement versus elicitation of effective resistance responses. Mol. Plant-Microbe Interact. 12:285-292.

Desvoyes, B., and Scholthof, H. B. 2002. Host-dependent recombination of Tomato bushy stunt virus coat protein mutant yields truncated capsid subunits that form virus-like complexes which benefit systemic spread. Virology 304:434-442.

Hearne, P. Q., Knorr, D. A., Hillman, B. I., and Morris, T. J. 1990. The complete genome structure and synthesis of infectious RNA from clones of tomato bushy stunt virus. Virology 177:141-151.

Hillman, B. I., Carrington, J. C., and Morris, T. J. 1987. A defective interfering RNA that contains a mosaic of a plant virus genome. Cell 51:427-433.

Hillman, B. I., Hearne, P., Rochon, D. A., Morris, T. J. 1989. Organization of tomato bushy stunt virus genome: Characterization of the coat protein gene and the $3^{\prime}$ terminus. Virology 169:42-50.

Johnston, J. C., and Rochon, D. M. 1990. Translation of cucumber necrosis virus RNA in vitro. J. Gen. Virol. 71:2233-2241.

Johnston, J. C., and Rochon, D. M. 1996. Both codon context and leader length contribute to efficient expression of two overlapping open reading frames of a cucumber necrosis virus bifunctional subgenomic mRNA. Virology 221:232-239.

Jones, R. W., Jackson, A. O., and Morris, T. J. 1990. Defective-interfering RNAs and elevated temperatures inhibit replication of tomato bushy stunt virus in inoculated protoplasts. Virology 176:539-545.

Knorr, D. A., Mullin, R. H., Hearne, P. Q., and Morris, T. J. 1991. De novo generation of defective interfering RNAs of tomato bushy stunt virus by high multiplicity passage. Virology 181:193-202.

Lee, U., Hong, J. S., Choi, J. K., Kim, K. C., Kim, Y. S., Curtis, I. S., Nam, H. G., and Lim, P. O. 2000. Broad bean wilt virus causes necrotic symptoms and generates defective RNAs in Capsicum annuum. Phytopathology 90:1390-1395.

Martelli, G. P., Gallitelli, D., and Russo, M. 1988. Tombusviruses. Pages 13-72 in: The Plant Viruses. Vol. 3. R. Koenig, ed. Plenum Publishing Corporation, New York.

Perrault, J. 1981. Origin and replication of defective interfering particles. Curr. Top. Microbiol. Immunol. 93:151-207.

Qiu, W., and Scholthof, H. B. 2001. Effects of inactivation of the coat protein and movement genes of Tomato bushy stunt virus on early accumulation of genomic and subgenomic RNAs. J. Gen. Virol. 82:3107-3114.

Qiu, W., Park, J.-W., Jackson, A. O., and Scholthof, H. B. 2001. Retention of a small replicase gene segment in Tomato bushy stunt virus defective RNAs inhibits their helper-mediated trans-accumulation. Virology 281:51-60.

Qiu, W., Park, J.-W., and Scholthof, H. B. 2002. Tombusvirus P19-mediated suppression of virus-induced gene silencing is controlled by genetic and dosage features that influence pathogenicity. Mol. PlantMicrobe. Interact. 15:269-280.

Ray, D., and White, K. A. 1999. Enhancer-like properties of an RNA element that modulates Tombusvirus RNA accumulation. Virology 256:162-171

Rezende, J. A. M., Garcia, S., and Scholthof, H. B. 1998. Tomato bushy stunt virus movement genes and host background affect the accumulation of defective interfering RNAs. Phytopathology 88:S75.

Rochon, D. M. 1991. Rapid de novo generation of defective interfering RNA by cucumber necrosis virus mutants that do not express the 20kDa nonstructural protein. Proc. Natl. Acad. Sci. U.S.A. 88:1115311157.

Rochon, D. M., and Johnston, J. C. 1991. Infectious transcripts from cloned cucumber necrosis virus cDNA: Evidence for a bifunctional subgenomic mRNA. Virology 181:656-665.

Roux, L., Simon, A. E., and Holland, J. J. 1991. Effects of defective interfering viruses on virus replication and pathogenesis. Adv. Virus Res. 40:181-211.

Russo, M., Burgyan, J., and Martelli, G. P. 1994. Molecular biology of Tombusviridae. Adv. Virus Res. 44:381-428

Sambrook, J., Fritsch, E. F., and Maniatis, T. 1989. Molecular Cloning: A Laboratory Manual, 2nd ed. Cold Spring Harbor Laboratory Press, Cold Spring Harbor, NY, U.S.A.

Scholthof, H. B., Desvoyes, B., Kuecker, J., and Whitehead, E. 1999. The biological activity of two tombusvirus proteins translated from nested genes is influenced by dosage control via context-dependent leaky scanning. Mol. Plant-Microbe Interact. 12:670-679.

Scholthof, H. B., Morris, T. J., and Jackson, A. O. 1993. The capsid protein gene of Tomato bushy stunt virus is dispensable for systemic move- 
ment and can be replaced for localized expression of foreign genes. Mol. Plant-Microbe Interact. 6:309-322.

Scholthof, H. B., and Jackson, A. O. 1997. The enigma of pX: A hostdependent cis-acting element with variable effects on tombusvirus RNA accumulation. Virology 237:56-65.

Scholthof, H. B., Scholthof, K.-B. G., and Jackson, A. O. 1995a. The tomato bushy stunt virus replicase proteins are coordinately expressed and membrane-associated. Virology 208:365-369.

Scholthof, H. B., Scholthof, K.-B. G., Kikkert, M., and Jackson, A. O. 1995b. Tomato bushy stunt virus spread is regulated by two nested genes that function in cell-to-cell movement and host-dependent systemic invasion. Virology 213:425-438.

Scholthof, K.-B. G., Scholthof, H. B., and Jackson, A. O. 1995c. Identification of tomato bushy stunt virus host-specific symptom determinants by expression of individual genes from a potato virus $\mathrm{X}$ vector. Plant Cell 7:1157-1172.

Scholthof, K.-B. G., Scholthof, H. B., and Jackson, A. O. 1995d. The effect of defective interfering RNAs on the accumulation of tomato bushy stunt virus proteins and implications for disease attenuation. Virology 211:324-328.

Szittya, G., Molnar, A., Silhavy, D., Hornyik, C., and Burgyan, J. 2002.
Short defective interfering RNAs of tombusviruses are not targeted but trigger post-transcriptional gene silencing against their helper virus. Plant Cell 14:359-372.

Szittya, G., Salamon, P., and Burgyan, J. 2000. The complete nucleotide sequence and synthesis of infectious RNA of genomic and defective interfering RNAs of TBSV-P. Virus Res. 69:131-136.

Turina, M., Omarov, R. Murphy, J. F., Bazaldua-Hernandez, C., Desvoyes, B., and Scholthof, H. B. 2003. A newly identified role for Tomato bushy stunt virus $\mathrm{P} 19$ in short distance spread. Mol. Plant Pathol. 4:67-72.

Voinnet, O., Pinto, Y. M., and Baulcombe, D. C. 1999. Suppression of gene silencing: A general strategy used by diverse DNA and RNA viruses of plants. Proc. Natl. Acad. Sci. U.S.A. 96:14147-14152.

White, K. A., and Morris, T. J. 1994. Nonhomologous RNA recombination in tombusviruses: Generation and evolution of defective interfering RNAs by stepwise deletions. J. Virol. 68:14-24.

White, K. A., and Morris, T. J. 1999. Defective and defective interfering RNAs of monopartite plus-strand RNA plant viruses. Curr. Top. Microbiol. Immunol. 239:1-17.

Wu, B., and White, K. A. 1998. Formation and amplification of a novel tombusvirus defective RNA which lacks the $5^{\prime}$ nontranslated region of the viral genome. J. Virol. 72:9897-9905. 